\title{
UN DIALOGUE AVEC SOI-MÊME ET LES AUTRES
}

Marges et marginalité dans Ping-pong. Version commentée, de Zviane

\section{A DIALOGUE WITH SELF AND OTHERS}

MARGINS AND MARGINALITY IN ZVIANE'S PING-PONG. VERSION COMMENTÉE

\section{UN DIÁLOGO CONSIGO MISMA Y LOS DEMÁS}

\section{MÁRGENES Y MARGINALIDAD EN PING-PONG.VERSION}

COMMENTÉE, DE ZVIANE

\section{CARMÉLIE JACOB}

Volume 43, numéro 2 (128), hiver 2018

La bande dessinée québécoise

URI : https://id.erudit.org/iderudit/1045067ar

DOI : https://doi.org/10.7202/1045067ar

Aller au sommaire du numéro

\section{Éditeur(s)}

Université du Québec à Montréal

\section{ISSN}

0318-9201 (imprimé)

1705-933X (numérique)

Découvrir la revue

Citer cet article

JACOB, C. (2018). UN DIALOGUE AVEC SOI-MÊME ET LES AUTRES : marges et marginalité dans Ping-pong. Version commentée, de Zviane. Voix et Images, 43(2), 95-111. https://doi.org/10.7202/1045067ar
Résumé de l'article

En 2014, Zviane publie à compte d'auteure Ping-Pong, un essai introspectif abordant les maillages entre les différentes pratiques artistiques. Une fois le tirage épuisé, les éditions Pow Pow proposent de rééditer l'essai, mais le format de la maison, plus large que celui de la bande dessinée originale, pose problème. Pour occuper l'espace laissé en trop sur chaque page, l'auteure choisit de commenter sa bande dessinée en plus d'inviter d'autres artistes québécois et français à faire de même, créant ainsi un " dialogue entre [elle] en 2014 et [elle] en 2015 ", en plus d'un " espace commun de réflexion où tout le monde se renvoie la balle » (p. 8 et rabat). Cette pratique met en lumière la difficulté de maintenir une cohérence avec soi-même dans la pratique de l'essai, qui, comme l'observe Pascal Riendeau, « exprime une pensée et déploie une subjectivité, mais [...] une pensée en mouvement, qui ne craint pas l'incomplétude " (Méditation et vision de l'essai, 2012, p. 11). L'autoréflexion et la remise en question, amenées par le dialogue avec les autres et les effets du passage du temps sur sa propre pensée, sont ainsi au coeur de Ping-Pong, et cet article analyse comment elles s'articulent au sein du texte comme du dessin. 


\section{UN DIALOGUE AVEC SOI-MÊME ET LES AUTRES}

Marges et marginalité dans

Ping-pong. Version commentée, de Zviane

$+++$

\section{CARMÉLIE JACOB}

Université du Québec à Montréal

En novembre 2014, Zviane autoéditait Ping-pong ${ }^{1}$, un essai bédéistique introspectif qui aborde principalement les maillages entre les différentes pratiques artistiques, et plus particulièrement, comme d'autres créations de la pianiste-bédéiste, les liens entre musique et bande dessinée. Imprimée en cinq cents exemplaires, l'œuvre a rapidement vu son premier tirage épuisé, bien qu'elle ait été publiée sous une licence Creative Common sans restriction, laquelle autorisait librement sa copie, sa modification et sa revente, en tout ou en partie. Devant ce succès, mais surtout mues par l'originalité de Ping-pong, les éditions Pow Pow ont rapidement proposé de rééditer le livre. Seul hic: le format de Pow Pow était sensiblement plus large que celui préconisé par Zviane pour ses planches, ce qui laissait d'importantes marges sur chaque page. Pour régler le problème, Luc Bossé, directeur de la maison d'édition, a proposé d'inviter un auteur à écrire dans les marges; mais Zviane a l'habitude de voir grand: «Pourquoi juste un invité2? » Après tout, l'œuvre avait, dans sa première forme, suscité de nombreuses réactions dans le milieu de la bande dessinée, et il ne serait pas difficile de trouver des auteurs qui accepteraient de prendre part au projet. La coordination d'une telle entreprise, toutefois, s'avérerait plus complexe: mieux valait que chaque auteur rédige une brève bande dessinée - qu'on placerait à la fin de l'ouvrage - en réaction à un élément du livre qui aurait retenu son attention. Et les marges, alors? "Écoute, y a plein d'affaires que j'ai écrites dans Ping-pong avec lesquelles je suis plus vraiment d'accord. Je pourrais utiliser les marges pour CHIALER ! [...] Pis en plus, ça prendrait la forme d'un dialogue entre moi en 2014 et moi en 2015... Un ping-pong dans le TEMPS!» ( $P$, 8; les majuscules sont dans le texte.) Zviane commentera donc sa propre œuvre moins de six mois après l'avoir publiée, et se servira aussi des marges pour renvoyer aux réactions des auteurs invités, placées en fin d'ouvrage.

1 Zviane [Sylvie-Anne Ménard], Ping-pong, Montréal, [s. é.] (publié à compte d'auteur), 2014, 147 p.

2 Zviane [et autres], Ping-pong. Version commentée, Montréal, Pow Pow, 2015, 226 p. Désormais, les références à cet ouvrage seront indiquées par le sigle $P$ suivi du folio, et placées entre parenthèses dans le texte. Nous remercions Luc Bossé, des éditions Pow Pow, de nous avoir aimablement transmis les fichiers d'impression des planches reproduites dans cet article. 
Le «ping-pong» du titre prendra ainsi un nouveau sens. Dans la mouture originale, il renvoie à un échange entre deux disciplines, idée qui prend forme lorsque l'auteure constate, après avoir observé plusieurs fois que la théorie musicale pouvait l'aider à comprendre la bande dessinée, que la théorie de la bande dessinée peut aussi l'amener à mieux comprendre la musique. Ainsi, une relation qui semblait à sens unique révélait son caractère réciproque («ça l'a rebondi pour la première fois» $[P, 21]$ ), ouvrant tout un champ de possibilités: s'il était possible de réfléchir aux interactions entre théorie musicale et théorie bédéistique, il était sans doute également possible de faire intervenir des notions de biologie en couture, de programmation en cuisine, etc., et inversement, de là le jeu de ping-pong entre les différentes disciplines. Ce jeu est maintenu dans Ping-pong. Version commentée, qui reprend toutes les planches de la première version, mais comprend aussi des annotations en vert qui montrent un jeu d'interactions entre Zviane en 2014 et Zviane en 2015, et entre Zviane et les dix-neuf bédéistes invités.

C'est à ces deux formes de jeu que nous réfléchirons ici, en nous intéressant plus spécifiquement au «hors-jeu», c'est-à-dire, d'abord, au caractère marginal de Pingpong, qui constitue l'un des rares essais d'introspection empruntant la forme bédéistique, puis à l'utilisation des marges elles-mêmes, laquelle élargit — au sens littéral — le terrain de l'essai. Nous verrons que le ping-pong proposé par Zviane repose sur une constante remise en question à la fois de la réflexion intellectuelle et de la pratique artistique, et que, bien qu'il soit extrêmement novateur dans le champ de la bande dessinée, il s'inscrit assez fidèlement à l'intérieur des limites de la définition de l'essai.

\section{JEU EN SIMPLE : L'INSCRIPTION DANS L'ESSAI}

La question du genre, en bande dessinée, est d'emblée complexe. Alors qu'on tend de plus en plus à considérer le média comme une forme, on y a longtemps vu un genre, paralittéraire qui plus est, et qui se serait lui-même ramifié en sous-genres (policier, comique, d'aventure, de science-fiction, etc.). Stéphanie Lamothe, qui a travaillé sur la bande dessinée d'autofiction au Québec, signale ainsi avec justesse qu' «une appellation pour la fiction n'est pas d'usage en bande dessinée ${ }^{3}$ », comme si la forme présupposait qu'il s'agissait de fiction. Ce préjugé peut s'expliquer par le fait que la critique, en cherchant à définir la bande dessinée, n'a pas pu résister à la tentation de hiérarchiser la présence de deux arts au service d'une même œuvre, et qu'elle a le plus souvent donné la préséance au dessin:

[L]e texte v[ient] au secours du dessin, et celui-ci n'[a] rien d'un simple «supplément» illustrant la parole. La chose se confirme si l'on songe qu'en plus des informations attributives d'ordre aspectuel (et non verbal), le dessin est aussi et surtout Action, c'est-à-dire support essentiel de ce genre d'«histoires». L'analogique l'emporte sur le discursif ${ }^{4}$.

3 Stéphanie Lamothe, Les modes d'expression du projet autobiographique dans la bande dessinée québécoise, mémoire de maîtrise, Montréal, Université du Québec à Montréal, 2011, f. 15.

4 Pierre Fresnault-Deruelle, «Le verbal dans les bandes dessinées», Communications, vol. XV, n 1, 1970, p. 156. 
Cherchant certainement à enrayer le préjugé selon lequel la bande dessinée, classée d'emblée dans le domaine littéraire plutôt que plastique en raison de son support (le livre), ne serait qu'un texte agrémenté d'images, Pierre Fresnault-Deruelle bascule rapidement vers l'autre extrême, qui n'est évidemment pas moins aprioriste. Le lecteur attentif objectera que Deruelle s'exprime à partir d'une Europe qui entame à peine la décennie 1970, alors que même le milieu underground étatsunien devra attendre encore deux ans avant la parution marquée des œuvres autobiographiques de Green, de Crump et de Spiegelman ${ }^{5}$. Pourtant, cette conception de la bande dessinée comme étant d'abord un média de l'image et, surtout, un média strictement narratif persiste, ainsi qu'en témoigne cette remarque de Pascal Robert tirée d'un ouvrage de 2016 :

La bande dessinée repose sur sa capacité à relâcher quatre paradoxes:

- raconter des histoires de manière privilégiée en images (sans forcément renier le texte cependant);

- raconter des histoires dynamiques avec des images fixes;

- raconter des histoires en images sur un support a priori privé de son;

- raconter des histoires en projetant un espace 3D sur un espace 2D (celui de la feuille), en privilégiant l'espace sur le temps ${ }^{6}$.

Pour Robert, la bande dessinée ne servirait donc qu'à «raconter des histoires», ce qui nous empêcherait de classer parmi les bandes dessinées non seulement Ping-pong, mais aussi, pour ne nommer que quelques exemples, le Bestiaire des fruits (2014) de la même auteure; Understanding Comics (1994), Reinventing Comics (2000) et Making Comics (2006) de Scott McCloud; In the Shadow of No Towers (2004) d'Art Spiegelman; Désœuvré (2008) de Lewis Trondheim; Economix (2012) de Michael Goodwin et Dan E. Burr; et The Influencing Machine (2012) de Brooke Gladstone et Josh Neufeld, sans compter les œuvres qui s'inscrivent dans le vaste champ du bédéreportage - auxquelles on pourrait prêter une valeur narrative dans la mesure où elles mettent souvent en scène le parcours du reporter, mais dont le caractère discursif reste indéniable.

Il ne s'agit pas de dire que l'essai occupe une place de choix en bande dessinée; sa présence est, au contraire, encore marginale. Nous remarquons seulement que la critique, malgré certains exemples connus, semble tenir à l'écart la pratique de l'essai, peut-être parce qu'elle complexifie l'exercice, déjà difficile, d'accoler une définition à la bande dessinée. Cet expédient se comprend d'autant mieux quand on considère que l'essai lui-même se prête mal à une définition, du fait que «les textes rangés dans la catégorie de l'essai lui sont la plupart du temps indexés par élimination des autres genres", cette catégorie regroupant finalement "tout ce qui reste lorsqu'on a éliminé la poésie et la fiction, narrative ou dramatique ${ }^{7} »$. Certains se sont néanmoins risqués

5 En outre, on sait qu'en France, le genre autobiographique ne commencera à s'imposer que dans les années 1990, principalement grâce aux maisons d'édition Ego comme X, le Simo, L'Association et Cornélius. Voir à ce sujet Laurence Croix, Le je des bulles, Rennes, Université Rennes 2-Haute-Bretagne, 1999, 122 f.

6 Pascal Robert, «Présentation générale. La bande dessinée numérique en questions... », Pascal Robert (dir.), Bande dessinée et numérique, Paris, CNRS éditions, coll. «Les essentiels d’Hermès», 2016, p. 15.

7 Pierre Glaudes et Jean-François Louette, L'essai, nouvelle édition revue et augmentée, Paris, Armand Colin, coll. «Lettres sup. Théorie des genres», 2011 [1999], p. 13. Les auteurs soulignent. 
à définir le genre en tenant compte de ses caractéristiques intrinsèques. Au Québec, la critique a particulièrement retenu la définition de Jean Marcel, qui a le mérite d'être succincte, mais surtout de n'être ni trop vague ni trop restrictive: «[L]'essai est la forme caractérisée de l'introduction dans le discours littéraire du JE comme générateur d'une réflexion de type lyrique sur un corpus culturel agissant comme médiateur entre les tensions fragmentaires de l'individualité dans sa relation à elle-même et au monde $^{8}$.» Cette définition comporte quatre éléments, soulignés par l'auteur, qui nous permettront de comprendre de quelle façon le Ping-pong de Zviane s'inscrit bel et bien dans ce genre. Nous proposons de les aborder à rebours.

D’abord, si le corpus culturel agit comme médiateur entre les tensions fragmentaires de l'individualité, il va de soi qu'il ne peut le faire que dans la mesure où l'auteur organise un parcours entre ces différentes tensions; comme le rappelle Jean Marcel, «l'objet culturel appréhendé par le JE-sujet de l'essai n'est lui-même que le produit d'une fragmentation du réel due aux conditions historiques et anthropologiques dans lesquelles il s'exerce», ce qui amène le JE à rechercher «sa propre unité en opposant à l'éclatement du réel un refus catégorique ${ }^{9} »$. Le défi que s'est donné Zviane avec Ping-pong tenait justement à ordonner cette médiation, à reconstituer si on veut le "casse-tête du réel». Après avoir pris conscience des interactions qui régissent l'ensemble des disciplines, l'auteure tente de tisser une toile qui révèle non seulement les différents enchevêtrements qui les unissent, mais ceux qui l'unissent, elle, à ces différentes disciplines. Comme Understanding Comics de Scott McCloud, Ping-pong se place de cette manière du côté de la sémiologie, sans pourtant jamais s'en revendiquer. Zviane pense ainsi le rapport à l'image, au langage et aux sons, passant tour à tour du signifié au signifiant, puis du signifiant au signifié, pour essayer de mieux comprendre ce qui se dresse entre l'individu et le monde dans lequel il évolue. L'une des principales entreprises de l'auteure est de «nommer les choses pour leur donner un ordre - mais aussi pour devenir plus sensible» $(P, 34)$; ainsi, elle se livre à de nombreuses réflexions sur le langage en général $(P, 45-48)$, s'interrogeant entre autres sur la nomenclature des sons $(P, 34-35)$, des éléments du design $(P$, 83-88), des composantes de la partition $(P, 89-93)$, des lignes du dessin $(P, 120-126)$. Ce qui l'intéresse, toutefois, ce qu'elle considère comme la véritable connaissance, ce n'est pas de «découvrir d[e] nouvelles étoiles; c'est [de] découvrir d[e] nouvelles constellations» $(P, 44)$, donc précisément de voir comment le corpus culturel permet la création de liens entre ce que Jean Marcel nomme les «tensions fragmentaires de l'individualité dans sa relation à elle-même et au monde».

Ce corpus culturel est dans Ping-pong extrêmement vaste compte tenu de la nature même du projet: tisser des liens entre les différentes disciplines. Si les arts plastiques et la musique ont la préséance - ce qui n'étonne pas dans l'œuvre d'une bédéiste et compositrice - l'informatique, la science, la danse, la cuisine et la psychologie sont aussi convoquées. Pour les aborder, Zviane recourt principalement à la citation et à l'anecdote, que Pascal Riendeau considère comme les «deux

8 Jean-Marcel Paquette, "Forme et fonction de l'essai dans la littérature espagnole», Études littéraires, vol. V, $\mathrm{n}^{\circ} 1$, avril 1972, p. 87. L'auteur souligne.

9 Ibid., p. 94. 
procédés littéraires principaux à partir desquels le discours essayistique établit généralement une stratégie argumentative ${ }^{10} »$; Ping-pong est éclaté dans sa forme, mais sa rhétorique reste celle de l'essai traditionnel. Pour étayer son argumentaire, Zviane convoque ainsi - parfois pour les réfuter - Archimède, Glenn Gould, Vincent d'Indy, Arthur Koestler, Daniel Levitin, Scott McCloud, Norman McLaren, Jackson Pollock, Oliver Sacks, Pierre Schaeffer et Chris Ware, en plus de nombreux collègues bédéistes, qu'on rencontre surtout au cœur d'anecdotes. Aguerrie depuis longtemps à la pratique du carnet, dont elle publiait les entrées sur son blogue ${ }^{11}$ avant de les faire paraître en recueils ${ }^{12}$, l'auteure est en effet passée maître dans l'art de l'anecdote. Celles qu'on retrouve dans Ping-pong diffèrent cependant de celles de ses carnets en ce qu'elles ne sont plus seulement factuelles, mais illustratives. En ce sens, comme l'a montré Marie-Pascale Huglo, elles s'inscrivent réellement dans une rhétorique persuasive:

Je considère l'anecdote en premier lieu comme un élément d'argumentation, comme un instrument apte à séduire plusieurs auditoires et à illustrer maintes thèses. L'illustration apparaît comme la vocation majeure de l'historiette. Pour n'être pas toujours un argument fiable, l'anecdote ne dispose pas moins d'un éventail persuasif étendu: amusante, curieuse, représentative, éclairante ou révélatrice, son capital à la fois didactique, divertissant et référentiel la dispose tout particulièrement à des fins illustratives ${ }^{13}$.

L'avantage du recours à l'anecdote, dans un essai en bande dessinée, est qu'elle n'est pas seulement apte à illustrer, mais apte à être illustrée. On prévient le lecteur sur le rabat de la première de couverture: Ping-pong renferme "vraiment vraiment beaucoup de texte», ce qui contredit l'affirmation de Fresnault-Deruelle selon laquelle l'analogique l'emporterait sur le discursif dans la bande dessinée. Cela dit, la forme exige une part d'analogique, et l'anecdote, grâce à son caractère narratif, plus facile à imager que le discours, permet son intégration. Du reste, la présentation divertissante de l'information est au centre des préoccupations de Zviane, comme en témoigne son projet d'un mémoire de maîtrise en composition musicale illustré de courtes bandes dessinées ${ }^{14}$.

10 Pascal Riendeau, Méditation et vision de l'essai. Roland Barthes, Milan Kundera et Jacques Brault, Québec, Nota bene, coll. «Littérature(s)», 2012, p. 41.

11 En ligne: http://www.zviane.com/prout (page consultée le 4 janvier 2018).

12 Zviane, La plus jolie fin du monde, Montrēal, Mécanique générale, 2007, 300 p.; et Le quart de millimètre, Longueuil, Grafigne.com éditions, coll. «Catapulte», 2009, 337 p. Les deux recueils ont été rassemblés sous le titre À l'école, publié aux éditions Mécanique générale (Montréal, 2016, 296 p.).

13 Marie-Pascale Huglo, Métamorphoses de l'insignifiant. Essai sur l'anecdote dans la modernité, Montréal, Balzac-Le Griot, coll. «L'univers des discours», 1997, p. 41.

14 «[P]ar souci littéraire, j'entends bien écrire un mémoire qui soit AGRÉABLE À LIRE. Ainsi le parsèmerai-je de courtes histoires graphiques afin d'égayer le lecteur, de dessins pour expliquer mes idées et d'apartés sous forme de petites $\mathrm{BD}$, qui viendront enfin mettre un terme à cette fâcheuse rumeur selon laquelle les mémoires de maîtrise en composition ne sont pas intéressants à faire ou bien intéressants à lire.» (Zviane, La plus jolie fin du monde, p. 225; les majuscules et l'italique sont de l'auteure.) Zviane abandonnera finalement sa maîtrise pour se consacrer à la bande dessinée, mais on rencontre un autre exemple de cet intérêt pour un accès divertissant à la connaissance dans le projet de site Web sur la théorie musicale qu'elle a 
La précision de Jean Marcel selon laquelle la réflexion essayistique devrait être «de type lyrique» est certainement l'élément de sa définition qui a le plus mystifié les chercheurs. En se basant sur Pensées, passions et proses, Riendeau souligne que

\begin{abstract}
Jean Marcel ne retient qu'un des trois éléments que l'on associe traditionnellement au lyrisme: sa musicalité. Selon lui, le discours de nature lyrique "s'évalue à la récurrence, au sein du texte, d'éléments formels spécifiquement linguistiques (phonétiques, lexicaux, syntaxiques ou sémantiques), dans la mesure où nous pouvons définir le lyrisme comme du langage qui, par son code propre, indique qu'il tend vers de la musique». L'auteur n'insiste donc pas sur les questions de subjectivité exacerbée et d'expression passionnée qu'on attribue aussi au lyrisme. [...] [C]e que la nature lyrique rappelle, c'est que l'essai, au sens entendu par Jean Marcel, s'affirme comme œuvre de littérature à part entière, dans laquelle la recherche d'une écriture se présente dans ses procédés littéraires dynamiques et variês ${ }^{15}$.
\end{abstract}

Si Riendeau ajoute que le lyrisme dont fait état Jean Marcel reste "une notion encombrante et difficile à conserver dans une analyse de tout type d'essai ${ }^{16}$ ", dans le cas de l'œuvre de Zviane, il s'avère intéressant à observer. Tout essai exige qu'on soigne sa forme, et Ping-pong innove justement sur ce plan, non pas tant en raison de sa valeur strictement littéraire que de sa configuration multiple. Le lyrisme, dans ce cas-ci, concerne l'organisation graphique autant que textuelle, et Zviane, en musicienne, réfléchit bien sûr à la question de la «musicalité» de ses bandes dessinées, qui est souvent évoquée lors d'entretiens. Remettant en question la définition de Vincent d'Indy selon laquelle «[1]a musique est l'art des sons» $(P, 49-51)$, elle en vient à la conclusion que «la musique est l'organisation du temps à l'aide de sons» ( $P$, 57; nous soulignons), et que c'est précisément ce qui permet à la poésie, à la danse, au dessin animé ou à la peinture d'être musicaux $(P, 58)$. Ainsi, «la bande dessinée peut être musicale puisqu' on peut la rythmer dans la grande forme, au chapitre, à la page, à la bande, à la case» $(P, 58)$, en recourant à l'espace pour représenter le temps. Le lyrisme de Ping-pong se construit donc dans un arrangement temporel du texte et du dessin, et passe aussi par des variantes typographiques auxquelles n'a pas accès l'auteur de l'essai traditionnel, dont la mise en forme est le plus souvent assurée par l'éditeur. L'une des qualités de la bande dessinée, selon Fresnault-Deruelle, est que le lecteur sait "sur quel ton sont prononcées les paroles des personnages ${ }^{17}$ ", qualité qu'on peut attribuer non seulement à l'expression des personnages, mais aussi à la typographie changeante: les lettres attachées chanteront doucement, tandis que les majuscules, le gras ou une taille plus grosse évoqueront le cri. En alliant au texte une typographie qui a priori ne lui convient pas, on pourra aussi connoter l'ironie,

mené avec la professeure Luce Beaudet, de l'Université de Montréal. Voir L'œil qui entend l'oreille qui voit. Un modèle d'analyse du discours harmonique tonal, en ligne: http://bw.musique.umontreal.ca/nm/apropos.htm (page consultée le 4 janvier 2018).

15 Pascal Riendeau, Méditation et vision de l'essai, p. 31-32. L'auteur souligne. La citation de Jean Marcel est tirée de Pensées, passions et proses, Montréal, l'Hexagone, coll. «Essais littéraires», 1992, p. 341.

16 Pascal Riendeau, Méditation et vision de l'essai, p. 32.

17 Pierre Fresnault-Deruelle, «Le verbal dans les bandes dessinées», p. 156. 
comme Zviane le fait en s'emportant contre un livre pour enfants $(P, 103 ;$ ill. 1$)$. Mentionnons au passage que l'auteure insère à plusieurs occasions des partitions dans ses bandes dessinées, y compris dans Ping-pong ( $P$, 32, 42, 44, 51, 56, 68, 74, 84, 90-91), lesquelles laissent évidemment entendre la musicalité au sens propre ${ }^{18}$.

\section{Désapprendre}

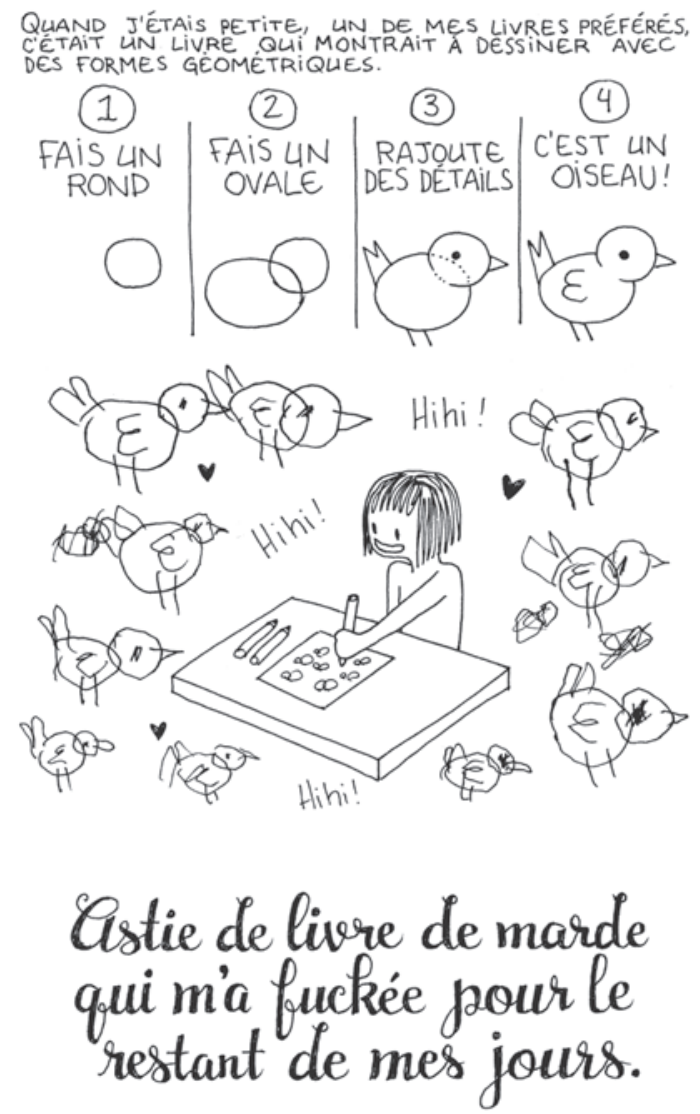

Ill. 1: Zviane, Ping-pong. Version commentée, p. 103.

18 L'introduction de partitions dans une œuvre littéraire n'est toutefois pas nouvelle; Balzac l'avait déjà prévue en 1844 dans Modeste mignon, bien que le projet ait finalement été contrecarré par des contraintes typographiques. Voir à ce sujet Michaël Ferrier, «Vie et usages de la marge. Littérature/musique: la "partition" ", Philippe Forest et Michelle Szkilnik (dir.), Théorie des marges littéraires, Nantes, Éditions Cécile Defaut/Université de Nantes, coll. «Horizons comparatistes», 2005, p. 237-253. 
Nous avons conservé pour la fin de cette démonstration le premier élément que Jean Marcel mentionne dans sa définition, à savoir que la réflexion essayistique est toujours générée par un «je», et est par conséquent nécessairement le fruit d'une subjectivité. En ce sens, l'essai partage certaines caractéristiques avec l'autofiction ou l'autobiographie, genre privilégié en bande dessinée et auquel Zviane s'est abondamment livrée dans ses carnets. Par exemple, Ping-pong pose un problème qu'a exploré Stéphanie Lamothe dans son mémoire sur la bande dessinée autobiographique québécoise: celui de «la cohabitation de deux registres expressifs [qui] entraine une réinterprétation des contraintes du pacte autobiographique ou de la stratégie d'ambiguïté ${ }^{19}{ }^{\prime}$; le «je» n'est plus seulement assumé par la parole, mais également par l'image, qui doit alors elle aussi répondre d'une stratégie d'autoreprésentation. Toutefois, contrairement à la bande dessinée autobiographique, qui peut se permettre, de façon à être le plus fidèle possible à ce que Philippe Lejeune a nommé le "pacte autobiographique ${ }^{20}$ ", de représenter son auteur de manière presque photographique ${ }^{21}$, l'essai doit éviter ce procédé, de peur de perdre son lecteur. Dans son essai Understanding Comics, Scott McCloud, après avoir tenté, pour une seule case, de troquer les traits épurés de son personnage pour un réalisme photographique, demande:

Would you have listened to me if I looked like this? I doubt it! You would have been far too aware of the messenger to fully receive the message. [...] I'm practically a blank slate! [...] You give me life by reading this book and by "filling up" this very iconic (cartoony) form. Who I am is irrelevant. I'm just a little piece of you. But if who I am matters less, maybe what I say will matter more 22 .

L'essai, malgré la posture subjective qu'il présuppose, exige une prise de distance par le «je» à l'égard de son propos, distance qui n'est pas aussi nécessaire dans le journal. Ce même phénomène contraint d'ailleurs Zviane à une autoreprésentation plus constante dans Ping-pong que dans ses carnets.

Ces derniers, on l'a dit, se rapprochent énormément du journal intime, en ce que leurs entrées sont datées et souvent fortement anecdotiques. Ils étaient du reste publiés sur le blogue de Zviane au fur et à mesure de leur rédaction, ce qui en garantit l'authenticité: impossible d'y revenir, de les modifier, de les supprimer sans rompre le "pacte» pris avec le lecteur ${ }^{23}$. Or, «le journal intime [...], en segmentant

19 Stêphanie Lamothe, Les modes d'expression du projet autobiographique dans la bande dessinée québécoise, f. 2.

20 Philippe Lejeune, Le pacte autobiographique, Paris, Éditions du Seuil, coll. «Poétique», 1975, 357 p.

21 C'est notamment la décision stylistique qu'a prise Fabrice Neaud dans son Journal (Angoulême, Ego comme X, 1996, 4 vol.).

22 «M'auriez-vous écouté si j'avais eu l'air de ça? J'en doute! Vous auriez été trop conscient du messager pour recevoir pleinement le message. [...] Je suis pratiquement un canevas! [...] Vous me faites exister en lisant ce livre et en "remplissant" la forme très iconique (illustrée) qui me représente. Il n'est pas important de savoir qui je suis. Je ne suis qu'une part de vous-même. Mais si la personne que je suis prend moins d'importance, le discours que je tiens en prendra peut-être davantage.» Scott McCloud, Understanding Comics. The Invisible Art, New York, Harper Collins, 1994, p. 36-37; nous traduisons.

23 On pourrait objecter que la présence d'un destinataire empêche l'association avec le journal intime, mais comme le note avec justesse Philippe Lejeune, «tout journal a un destinataire, ne serait-ce que soi-même 
l'énonciation, [...] rapproche l'événement de son compte rendu et de son commentaire ${ }^{24}$ ", ce qui permet certains jeux graphiques qui ne sont pas autorisés par l'essai. Ainsi, dans les carnets, le style de dessin change fréquemment au gré des émotions de l'auteure. L'entrée du 18 septembre $2006^{25}$, par exemple, commence dans le style alors propre à la bédéiste, pour se terminer par un trait excessivement épuré, presque enfantin, qui montre que les deux dernières planches ont été réalisées rapidement et sous le coup de la frustration - les expressions «Fuck you!», "perte de temps» et «néant ${ }^{26}$ » attirent l'œil. Cette stratégie rhétorique n'est rendue possible que par la quasi-concordance entre le temps de l'énonciation et le temps diégétique qui est le propre du journal. L'essai, par contre, suppose une organisation rationnelle du discours, qui s'opère a posteriori. Alors que l'autofiction repose sur un pacte d'authenticité, et la fiction, sur ce que Pierre Glaudes et Jean-François Louette appellent un " "pacte de mauvaise foi" en vertu duquel chacun "fait semblant" 27 ", l'essai ne garantit pour sa part que la sincérité. Ainsi son auteur «met en avant sinon sa capacité à prouver qu'il dit vrai, du moins sa volonté de ne rien dissimuler des limites de ses investigations, au nom de son exigence de vérité28 ». C'est cette dernière exigence qui rend intéressante l'occasion qu'a donnée à Zviane la réédition de Ping-pong, laquelle lui permettait de revenir, après quelques mois, sur une œuvre qu'elle avait livrée suivant non pas une exigence de vérité, comme dans ses carnets, mais une exigence de sincérité.

\section{JEU EN DOUBLE : LES INSCRIPTIONS DANS LES MARGES DE L'ESSAI}

Partant de la définition de la sincérité, Philippe Gasparini souligne qu'on la présente sémantiquement comme le fait d'une «triple intention: de discrimination, de déclaration franche, et de refus du leurre ${ }^{29}$ ". Elle ne présume pas d'un désir de "tout dire», ce qui était le projet de Rousseau dans ses Confessions ${ }^{30}$, mais bien de raconter, sans mentir, ce qui aura retenu l'attention comme étant pertinent. La tâche de l'essayiste est donc de rapporter fidèlement les preuves de son argumentaire, qui sont principalement les citations et les anecdotes dont nous avons parlé plus haut, et de les analyser selon ce qu'il croit au moment de l'énonciation. Comme l'observe Riendeau, «l'essai exprime une pensée et déploie une subjectivité, mais c'est une pensée en mouvement, qui ne craint pas l'incomplétude ${ }^{31} »$. Ainsi, l'essayiste pourra sans cesse

plus tard», et est par conséquent régi par la même forme de pacte que les autres formes autobiographiques. Philippe Lejeune, Signes de vie. Le pacte autobiographique 2, Paris, Éditions du Seuil, 2005, p. 27.

24 Philippe Gasparini, Est-il je? Roman autobiographique et autofiction, Paris, Éditions du Seuil, coll. «Poétique», 2004, p. 186.

25 Zviane, «Adieu la compo! Vive la compo!», La plus jolie fin du monde, p. 151-157.

26 Ibid., p. 157-158.

27 Pierre Glaudes et Jean-François Louette, L'essai, p. 36.

28 Ibid., p. 36-37.

29 Philippe Gasparini, Est-il je?, p. 233.

30 Voir à ce sujet le chapitre «Tout dire» dans Philippe Lejeune, Signes de vie, p. 201-207.

31 Pascal Riendeau, Méditation et vision de l'essai, p. 11. 
revenir sur ce qu'il a dit, par exemple pour ajouter ce qu'il avait oublié, ce qu'il ne savait pas ou ce à quoi il ne pensait pas au moment de l'énonciation. Il est d'ailleurs assez commun que les essais subissent, au fil de leurs rééditions, des modifications - on pense aux thèses publiées et aux divers travaux savants, mais Montaigne, qu'on associe à la genèse du genre, adoptait déjà cette pratique ${ }^{32}-$, alors que la chose est rare avec les œuvres de fiction ${ }^{33}$, qui peuvent poser un problème esthétique, mais ne font pas intervenir l'exigence de sincérité.

Dans Ping-pong. Version commentée, le désir de rectifier certaines idées, à la lumière d'une réflexion nouvelle, est ce qui justifie a priori l'utilisation des marges. Le projet s'y prêtait d'autant mieux que, dans les premières pages de l'essai original, où l'auteure explique sa démarche, on lit: "Y'a rien qui a la prétention d'être une vérité; c'est juste des pistes de réflexion vagues, avec lesquelles je serai probablement en désaccord dans une couple d'années.» Cette affirmation invite évidemment l'auteure $^{34}$ à un commentaire en marge de la réédition: "Ça fait même pas six mois et je suis déjà en désaccord avec un paquet d'affaires.» $(P, 22)$ La version commentée l'amène donc à revenir, avec un peu de recul, sur les idées qu'elle avait exprimées. Par exemple, en 2015, elle conteste une proposition à laquelle elle adhérait en 2014 : «L'analyse, c'est le sensible!» ( $P$, 93; l'auteure souligne), et répond: "Alors là, NON! L'analyse n'est pas le sensible. L'analyse arrive après la sensation. Le vrai observateur, c'est celui qui observe sans analyser. Sinon, ce n'est pas un observateur, $c^{\prime}$ est un interprète.» $(P, 93$; les majuscules sont dans le texte.) Dans ce cas-ci, la rétractation est aussi péremptoire que l'affirmation initiale. Certains passages, toutefois, montrent que le processus d'annotation amène avec lui la conscience d'une constante remise en question. C'est notamment le cas dans le chapitre «Jaune n'est pas jaune», où Zviane se contente de remarquer que certains termes mériteraient

32 Michel Hansen fait remarquer à ce sujet que l'essai «Nous ne goûtons rien de pur», qui «dans l'édition de 1580 fait à peine une page», subit au fil du temps des ajouts qui «vont multiplier par 6 la masse [du texte] et, ce faisant, retourner son message». Michel Hansen, «Des marges du savoir aux savoirs de la marge. La marge dans les Essais de Montaigne», François Marotin (dir.), La marge. Actes du colloque de ClermontFerrand (janvier 1986), Clermont-Ferrand, Association des Publications de la Faculté des lettres et sciences humaines de Clermont-Ferrand, 1988, p. 91.

33 À titre de contre-exemple, on pensera à l'entreprise de réécriture à laquelle s'est livré Dany Laferrière, par exemple avec Le goût des jeunes filles ou Cette grenade dans la main du jeune Nègre est-elle une arme ou un fruit?, mais les œuvres de Laferrière, autofictionnelles et fortement introspectives, sont à mi-chemin entre l'œuvre de fiction et l'essai.

34 Compte tenu de l'inscription de Ping-pong dans l'essai, nous ne ferons pas de distinction entre ce qu'on aurait pu appeler le "personnage» de Zviane et l'auteure elle-même. Riendeau rappelle que, dans l'essai, «le "je" textuel mis en scène ne possède pas un statut toujours clair: référentiel pour certains, strictement fictionnel pour d'autres, il crée une véritable ambivalence. Il semble pourtant entendu que l'essayiste parle en son nom et qu'il devient même abusif de parler de narrateur pour désigner celui qui remplit le rôle d'instance énonciative du discours essayistique» (Méditation et vision de l'essai, p. 32). Or, dans Ping-pong, un problème supplémentaire se pose, qui est celui du personnage. Le discours essayistique est le plus souvent donné sous la forme d'un long texte encadré placé dans le haut de l'image. À l'occasion, toutefois, ce long texte apparaît dans un phylactère relié à une autoreprésentation de Zviane. Il serait tentant de voir un personnage dans cette autoreprésentation, mais comme le texte "prononcé» s'inscrit généralement dans la suite logique du texte encadré, la scission deviendrait rapidement problématique. Pour les besoins de cet article, nous avons choisi de contourner le problème, qui mériterait de faire l'objet d'une étude à part entière. 
d'être explicités et que des questions qu'elle abordait rapidement sont plus complexes qu'elle se l'était imaginé au premier abord. Sur la même page, après avoir réfuté une affirmation ("“On ne peut penser que par le langage?" COME ON! Est-ce que j'ai besoin du langage quand j'apprends à monter à vélo?»), elle utilise la marge du bas pour inscrire un timide «(ben peut-être, en fait)» $(P, 42 ;$ ill. 2$)$, révélant que c'est bien un dialogue qui s'instaure entre la Zviane de 2014 et celle de 2015, et que cette dernière, malgré sa "séniorité», n'a pas beaucoup plus d'autorité ni de certitudes que la première. Ainsi, elle recourt à l'annotation d'une façon contraire à celle qu'on voit le plus souvent, qui consiste à gloser le texte pour en donner une explication. François Marotin dit de la marge que, «dans la mesure où elle cherche à éviter les incompréhensions, à lever les ambiguïtés, elle restreint le sens du texte riche de tous ses possibles», ajoutant que "[l]'auteur lui-même risque d'affadir son œuvre lorsqu'il réfléchit en marge de son texte ${ }^{35}$ ». C'est vrai lorsque l'auteur, pris de panique à l'ídée que son lecteur passe à côté de ce qu'il aura eu tant de mal à inscrire dans son œuvre, s'affaire à lui en donner toutes les clefs. Zviane recourt plutôt aux marges pour montrer qu'elle-même ne possède pas nécessairement ces clefs, que le lecteur sera peut-être en mesure de lui fournir.

L'intérêt de la version commentée de Ping-pong est qu'elle met réellement en valeur les échanges suggérés par le titre. Si Michel Hansen remarque que «le problème de la marge est étroitement lié à celui de l'intertextualitée ${ }^{36}$ ", il faut admettre que c'est le cas de l'essai en général, qui repose abondamment sur la citation et le recours à des connaissances diverses. La version originale de Ping-pong n'était ainsi pas dépourvue d'échanges, mais ceux-ci avaient lieu entre différents penseurs et chercheurs, issus de domaines divers, que Zviane faisait se confronter. La version commentée tient au contraire réellement du dialogue. Si le projet initial de Zviane de faire intervenir ses contemporains dans les marges s'avère trop complexe d'un point de vue éditorial, la formule choisie permet tout de même à des bédéistes de se prononcer sur certaines choses ou de partager leur expérience, la marge servant alors à effectuer un renvoi à leurs interventions (voir $P, 127$ et 215; ill. 3 et 4). La version commentée se présente ainsi comme un semi-collectif: sur la première de couverture et le dos, seul figure le nom de Zviane; sur la quatrième de couverture, toutefois, apparaissent les noms des dix-neuf bédéistes qui ont commenté l'œuvre. Le graphisme simple, illustrant en vert sur fond jaune la trajectoire d'une balle de ping-pong, met en valeur les noms des auteurs qui ont participé et révèle l'échange qui aura lieu entre eux et Zviane.

En cela, Ping-pong. Version commentée affiche d'emblée un projet différent de celui autoédité par Zviane, dont la couverture insistait sur l'individualité et l'interdisciplinarité en présentant de multiples autoreprésentations de Zviane s'affairant à la couture, au piano, à la cuisine, au dessin ou à la lecture. Cette couverture était divisée à la manière d'une table de ping-pong, la ligne blanche traçant une distinction claire entre l'entrain et le découragement, insistant sur les différentes facettes d'un même individu. Le seul élément propre aux deux couvertures est la

35 François Marotin, «Introduction», François Marotin (dir.), La marge, p. x.

36 Michel Hansen, «Des marges du savoir aux savoirs de la marge», p. 90. 


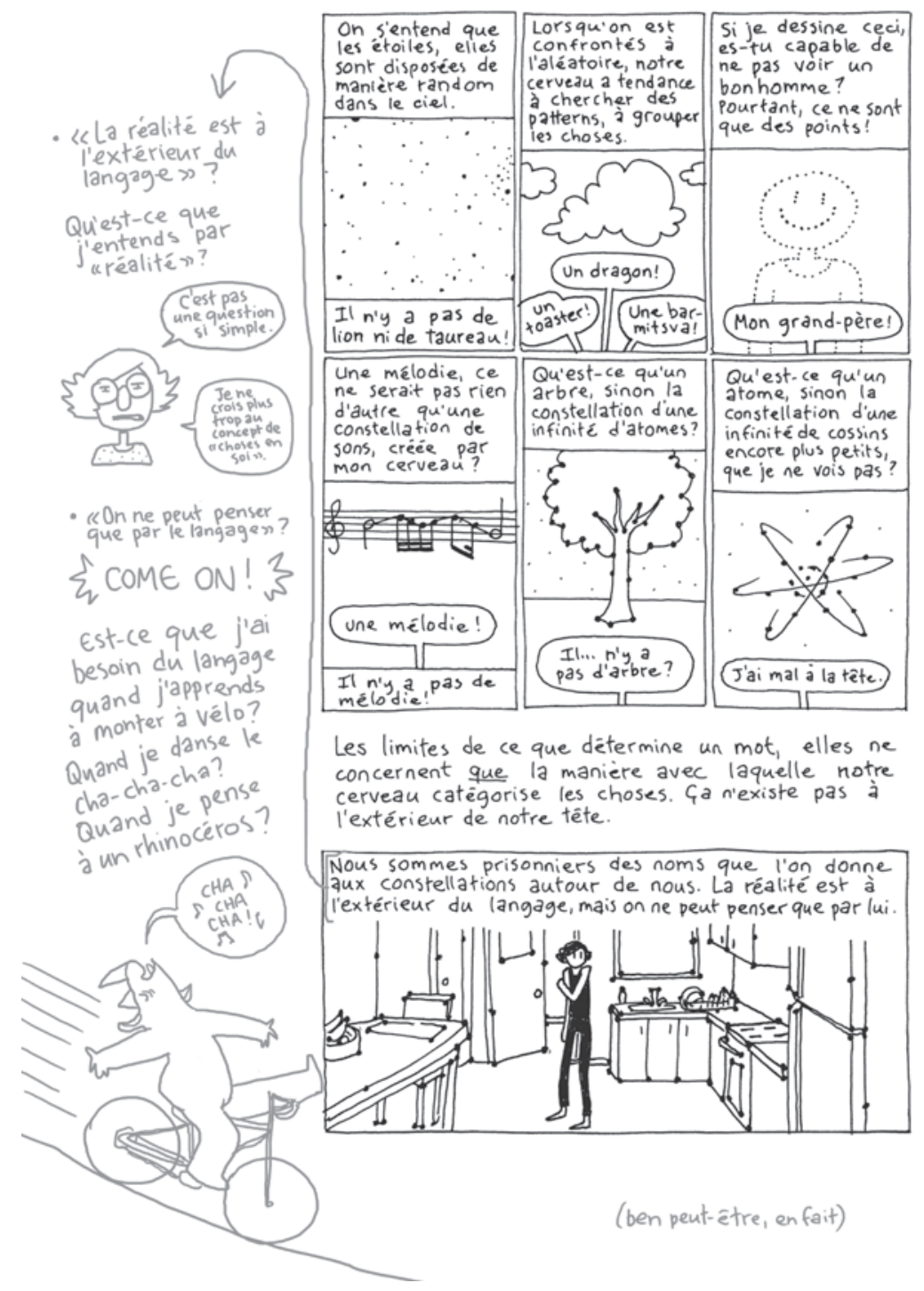

Ill. 2 : Zviane, Ping-pong. Version commentée, p. 42.

représentation de la balle et de sa trajectoire, mais cette fois, la balle rebondit sur la tête de Zviane. Aucune équivoque possible: l'échange, dans le Ping-pong original, est purement intellectuel et individuel, l'intertextualité étant à tout moment orientée par l'essayiste. Si Riendeau rappelle que «citer, c'est en quelque sorte accueillir l'autre, lui donner la parole», il admet aussi que «l'essayiste s'autorise, jusqu'à un certain point, à prendre la parole en allant la chercher chez l'autre, en créant un 


\section{Croiser les choses}

Ces pages ont été dessinées en 20ll, soit 3 ans avant le reste du

Arthur Koestler est un cool.

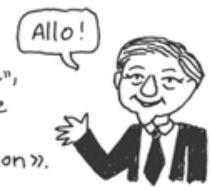
livre.

Dans son bouquin "Le cri d'Archimède" il tisse un lien entre humour, poésie et découverte scientifique.

Pour ça, il invente le mot "bissociation".
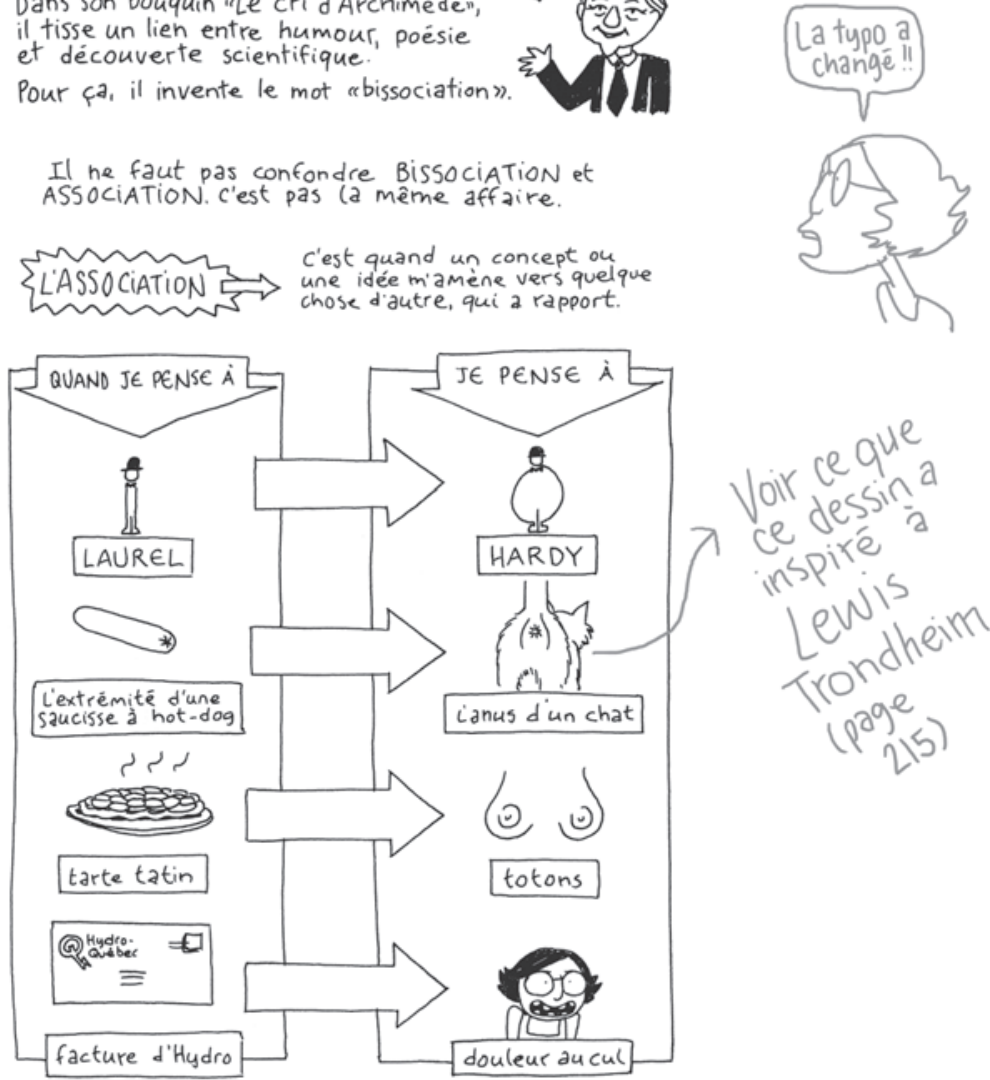

Ill. 3: Zviane, Ping-pong. Version commentée, p. 127.

rapport dialogique complexe et en certains cas conflictuel avec un autre discours ${ }^{37}$ ». En accueillant littéralement l'autre dans les pages de son œuvre, Zviane renonce à s'imposer dans cette part du processus intertextuel.

37 Pascal Riendeau, Méditation et vision de l'essai, p. 43. 


\section{Le commentaire de}

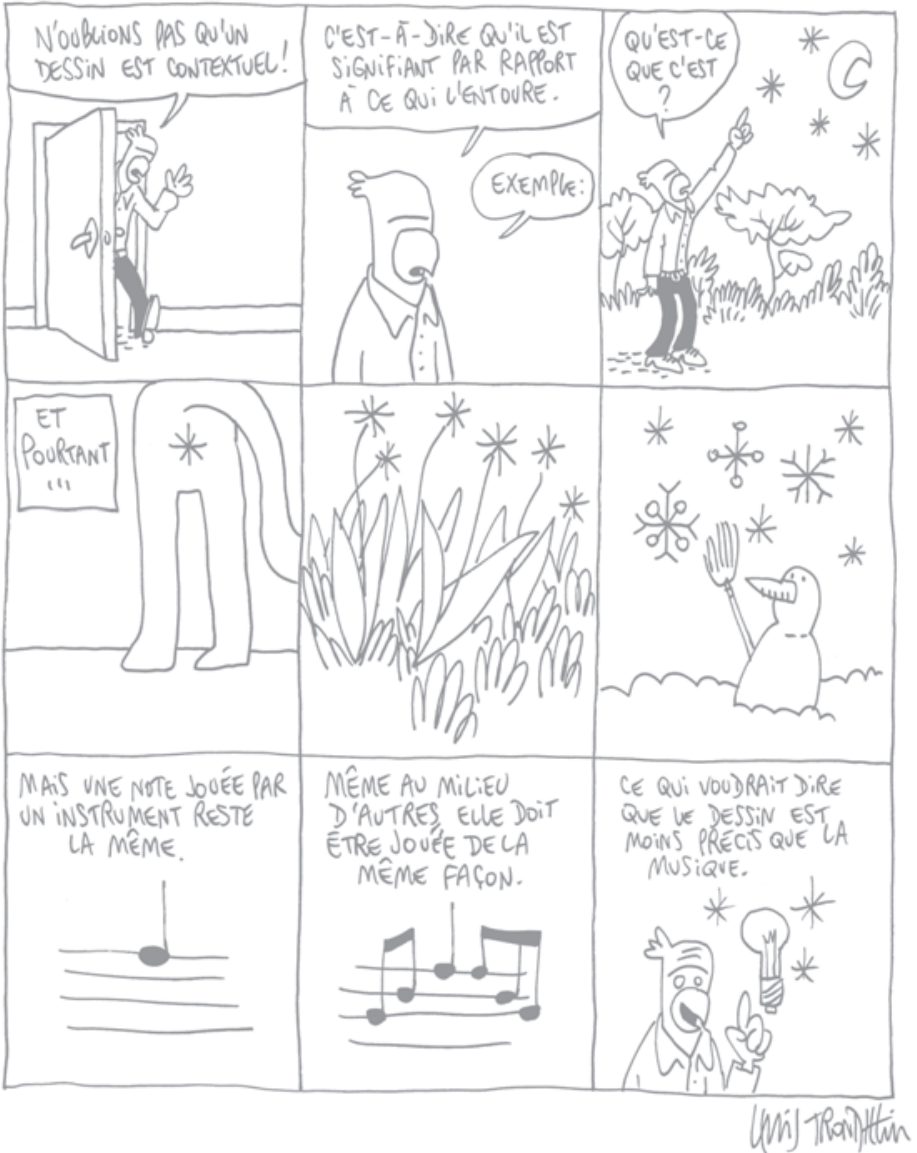

Ill. 4: Zviane, Ping-pong. Version commentée, p. 215.

Hormis pour la couverture, les crédits éditoriaux et quelques erreurs grammaticales ou lexicales, la version commentée n'effectue aucune modification à celle publiée à compte d'auteur. On n'y trouve que des ajouts, indiqués en vert. Ces ajouts peuvent être des ratures ou des «X»; il n'en reste pas moins que la version originale est en tout temps accessible et assumée comme le témoin d'une pensée et 
d'une démarche artistique qui ont évolué au fil du temps. Ainsi, alors que le passage d'une publication à compte d'auteur à une publication en maison d'édition exige généralement de l'auteur qu'il travaille avec une direction littéraire afin d'apporter à son texte des modifications qui en amélioreront la qualité, ce protocole est, dans Ping-pong. Version commentée, révélé plutôt qu'appliqué, comme en témoigne une discussion entre Zviane et Luc Bossé, dont la conclusion est simplement: «OK C’EST UN DESSIN DE MARDE.» ( $P$, 100; ill. 5; les majuscules sont dans le texte.)

À un autre endroit, Zviane écrit en marge qu'elle «aurai[t] donc dû prendre trois pages au lieu de juste une pour expliquer ça» et conclut que le chapitre qui se termine "est incomplet et [qu']il n'est pas super clair» $(P, 43-44)$. Ce faisant, elle met en lumière l'importance de l'organisation textuelle et du travail artistique qui s'opère généralement dans la solitude de l'auteur et dont les détails ne sont habituellement pas livrés au lecteur. La marge devient le lieu de la réflexion personnelle, telle qu'elle l'est - ou du moins l'était avant l'avènement du traitement de texte - au moment du travail créateur. Comme l'exprime bien Régine Jomand-Beaudry, toutefois, la marge sert aussi à «mettre à mal le postulat de l'immutabilité du texte littéraire, celui de son autosuffisance et de son unité ${ }^{38}$ ", fonction dont Zviane se sert lorsque les modifications auxquelles elle pense n'exigent pas de changements structuraux ou esthétiques importants. Ainsi, dans le chapitre "Désapprendre», elle raye un mot («créant») pour le remplacer par un autre («reproduisant») et se permet même $\mathrm{d}^{\prime}$ «ajout[er] un peu de contraste à [une] image pour la rendre plus lisible» $(P, 118$ 119), exploitant le champ de possibilités que lui ouvre l'utilisation du vert dans une œuvre qui n'était originalement qu'imprimée en noir.

Qu'il s'agisse de la version originale ou de la version commentée, ce que remarque Christine Noille-Clauzade s'applique: «[O]n ne finit pas par la spécification des marges dans un travail de peinture, d'imprimerie, de traitement de texte informatique, mais on commence par là ${ }^{39} . »$ Ainsi, le projet initial de Zviane était circonscrit dans un format d'environ 7 po sur 4,5 po; celui de la réédition dans un format de 8,25 po sur 6,35 po, et dans les deux cas, tout le processus artistique a été pensé en fonction de la marge, soit en raison de son caractère contraignant, soit au contraire pour le nouvel espace de liberté qu'elle offrait. Jan Baetens rappelle toutefois que la bande dessinée autorise que «le monde diégétique s'empare du niveau "supérieur", narratologiquement parlant, des blancs et du supercadre ${ }^{40}$ » - autrement dit, que le bédéiste a le pouvoir de transgresser les frontières imposées par les marges. Zviane se prêtait déjà au jeu dans la première version de Ping-pong en recourant à ce qu' on appelle en imprimerie une «marge perdue» ou un «bleed», pour exprimer la vastitude indéterminée des «choses qu['elle] sai[t] pas qu['elle] sai[t] pas» $(P, 138-139)$, représentée par un cercle dont les limites s'étendent à l'extérieur de la page. Elle recourt au même procédé dans Ping-pong. Version commentée, sans

38 Régine Jomand-Beaudry, «Pour une théorie des marges littéraires», Philippe Forest et Michelle Szkilnik (dir.), Théorie des marges littéraires, p. 20.

39 Christine Noille-Clauzade, «Rhétoriques de la mise en marge», Philippe Forest et Michelle Szkilnik (dir.), Théorie des marges littéraires, p. 51.

40 Jan Baetens, «Pour une poétique de la gouttière», Word \& Image, vol. VII, n 4, octobre-décembre 1991, p. 374. 


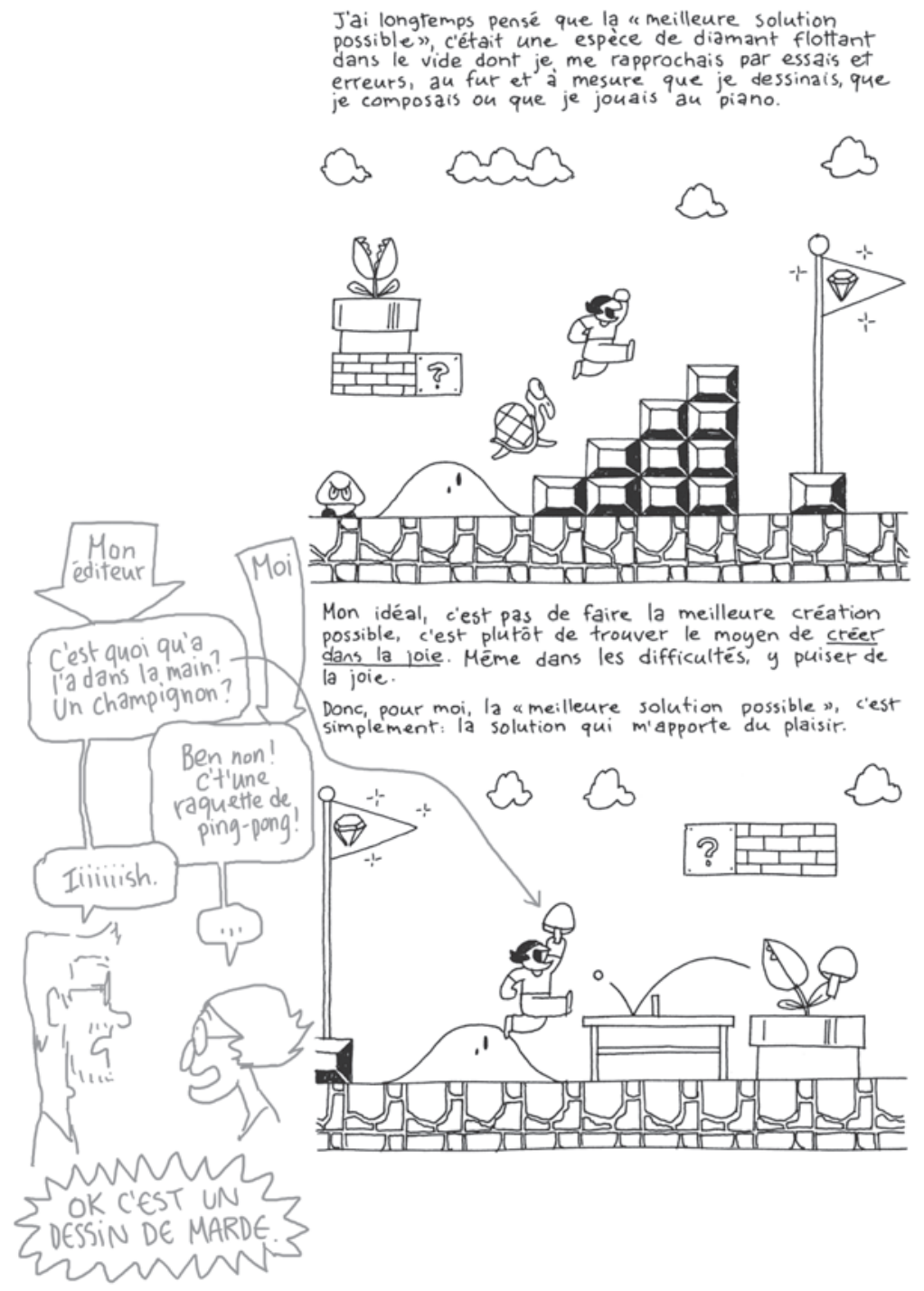

Ill. 5: Zviane, Ping-pong. Version commentée, p. 100.

utiliser le vert pour la section qui dépasse des marges initiales - comme il était sousentendu que les marges s'étendaient bien au-delà du livre, il n'y a après tout aucune tricherie à les prolonger dans cette deuxième édition -, mais elle se permet aussi par exemple d'ajouter une case à la première version d'une planche $(P, 137)$. Ce faisant, Zviane se sert des marges pour repousser sciemment les limites de la planche. Si la 
marge, comme l'explique Noille-Clauzade, «contribue à une reconceptualisation du texte, à une contestation de sa clôture, à sa compréhension en concept "ouvert" 41 ", Ping-pong. Version commentée en est l'illustration parfaite, puisque y concordent ouverture spatiale et ouverture intellectuelle.

Le premier Ping-pong de Zviane, publié à compte d'auteur, était intéressant en ce qu'il s'inscrivait dans le genre de l'essai, ce qui est encore marginal dans le domaine de la bande dessinée, fortement associée à la fiction et à la narrativité. Ce l'est plus encore lorsqu'il s'agit d'un essai introspectif, qui répond sur tous les plans à la définition qu'a proposée Jean Marcel; des bandes dessinées comme Economix ou The Influencing Machine, par exemple, sont des essais "par élimination", au sens où, en tant qu'ouvrages de vulgarisation, ils ne peuvent être classifiés ni parmi les textes de fiction ni parmi les œuvres poétiques. Par contre, la subjectivité y transparaît assez peu, pour faire principalement place à une perspective historique, facilement narrativisable. À l'opposé, une œuvre comme Paying for it de Chester Brown, qui réfléchit à la décriminalisation de la prostitution, octroie une place importante à la subjectivité, mais l'appareil critique est presque entièrement relégué en notes de fin d'ouvrage, de sorte que la bande dessinée elle-même peut difficilement être considérée comme un essai. Au contraire, avec Ping-pong, Zviane est parvenue à inscrire la discursivité à même l'ouvrage, projet que Fresnault-Deruelle, en 1970, semblait considérer comme impossible. L'intérêt principal de l'œuvre réside toutefois dans sa version commentée, publiée chez Pow Pow, laquelle élargit la polysémie du titre pour évoquer des échanges qui ne se font plus seulement entre les idées et les disciplines, mais entre des bédéistes contemporains, et entre deux âges de la même auteure. La décision artistique qui, au départ, était mue par une contrainte éditoriale laisse transparaître les traces du processus de création et de l'évolution d'une même pensée; ainsi, l'essayiste peut revenir sur ses dires d'une façon que peu de philosophes auraient récusée, et qui n'est certainement pas étrangère à la pratique du blogue à laquelle elle s'est longtemps livrée.

41 Christine Noille-Clauzade, «Rhétoriques de la mise en marge», p. 40-41. 